\title{
COMUNICAÇÃO
}

\section{ABELHAS (Hymenoptera: Apoidea) VISITANTES DAS FLORES DE GLIRICÍDIA NO RECÔNCAVO BAIANO}

\author{
Bees (Hymenoptera: Apoidea) visitors of gliricidia flowers \\ (Gliricidia sepium (Jacq.) Stend.) in the Reconcavo Baiano region
}

\author{
Carlos Alfredo Lopes de Carvalho', Gabriela Andrade de Oliveira², Mayana Matos Oliveira ${ }^{3}$, \\ Ygor Pereira de Sant'anna ${ }^{3}$, Cerilene Santiago Machado ${ }^{4}$
}

\begin{abstract}
RESUMO
Objetivou-se, nesse trabalho, obter informações sobre a diversidade de abelhas visitantes em gliricídia (Gliricidia sepium (Jacq.) Stend.), na região do Recôncavo Baiano. As espécies visitantes nas flores foram coletadas, no período de setembro a novembro de 2005. Definiu-se, aleatoriamente, 15 inflorescências por intervalo de hora para a coleta efetiva das abelhas, sendo utilizado um minuto por inflorescência, ao longo do intervalo de 07:00 às 18:00 horas, durante o período de floração. Um total de 10 espécies foram identificadas, sendo que Apis mellifera foi a espécie mais abundante, com freqüência relativa igual a 25,40\%, seguida da Trigona spinipes (23,81\%), Nannotrigona testaceicornis (14,28 \%), Xylocopa grisescens e Trigona fuscipennis (ambas com $12,70 \%$ ). O pico de visita das abelhas ocorreu das 10:01 às 11:00 horas. A. mellifera, $T$. spinipes e $N$. testaceicornis foram consideradas as espécies com potencial para a polinização das flores de gliricídias, na região do Recôncavo Baiano.
\end{abstract}

Termos para indexação: Insecta, Gliricidia sepium, abelhas sem-ferrão, Meliponinae.

\section{ABSTRACT}

The focus of this paper was to get information about the diversity of bees visiting Gliricidia sepium (Jacq.) Stend. in the Recôncavo Baiano region. The species visiting the flowers were collected in the period from September to November 2005. For the collection of the bees, 15 inflorescences were defined randomly per hour, one minute being used per inflorescence, throughout the interval from 07:00 a.m. to 06:00 p.m., during the blooming period. A total of ten species were identified, Apis mellifera being the most abundant species, with relative frequency equal to $25.40 \%$, followed by Trigona spinipes (23.81\%), Nannotrigona testaceicornis (14.28\%), Xylocopa grisescens, and Trigona fuscipennis (both with 12.70\%). The peak of bee visits occurred from 10:01a.m. to 11:00 a.m. A. mellifera, T. spinipes, and $N$. testaceicornis were considered the species with potential for the pollination of Gliciridia sepium flowers in the region of the Recôncavo Baiano.

Index terms: Insecta, Gliricidia sepium, stingless bees, Meliponinae

\section{(Recebido em 10 de agosto de 2006 e aprovado em 1 de abril de 2008)}

A Gliricidia sepium (Jacq.) Steud. é uma planta da família Fabaceae distribuída em regiões tropicais, nativa da América do Sul e Central (SUMBERG, 1985), de grande interesse comercial e econômico para regiões tropicais pelas suas características de múltiplo uso (DRUMOND \& CARVALHO FILHO, 2006).

No Nordeste brasileiro, há muitos anos, essa espécie é cultivada na região cacaueira da Bahia, para o sombreamento do cacau, tendo sido recentemente introduzida nos estados de Pernambuco e Sergipe (DRUMOND \& CARVALHO FILHO, 2006). A gliricídia não tem nome vulgar no Brasil, porém nos países de língua espanhola é chamada de madre-de-cacao, mata-ratón e madero-negro (DRUMOND \& CARVALHO FILHO, 2006; KIILL \& DRUMOND, 2001; NEVES, 2006).

As flores da gliricídia são reunidas em inflorescências terminais, do tipo cacho ou racemo (DRUMOND \& CARVALHO FILHO, 2006). Kiill \& Drumond

\footnotetext{
${ }^{1}$ Engenheiro Agrônomo, Doutor, Professor Adjunto - Centro de Ciências Agrárias, Ambientais e Biológicas/CCAAB - Universidade Federal do Recôncavo da Bahia/UFRB - Insecta - Cx. P. 118 - 44380-000 - Cruz das Almas, BA - calfredo@ufrb.edu.br - Bolsista PQ-CNPq

${ }^{2}$ Graduanda em Agronomia - Centro de Ciências Agrárias, Ambientais e Biológicas/CCAAB - Universidade Federal do Recôncavo da Bahia/UFRB Insecta - Cx. P. 118 - 44380-000 - Cruz das Almas, BA - gabyufba@gmail.com - Bolsista PIBIC-FAPESB

${ }^{3}$ Graduandos em Agronomia - Centro de Ciências Agrárias, Ambientais e Biológicas/CCAAB - Universidade Federal do Recôncavo da Bahia/UFRB Insecta - Cx. P. 118 - 44380-000 - Cruz das Almas, BA - mayanamatos@ig.com.br

${ }^{4}$ Engenheira Agrônoma, Mestre - Centro de Ciências Agrárias, Ambientais e Biológicas/CCAAB - Universidade Federal do Recôncavo da Bahia/UFRB Insecta - Cx. P. 118 - 44380-000 - Cruz das Almas, BA - cerilenes@yahoo.com.br - Bolsista CNPq
} 
(2001), estudando a sua biologia floral, observaram que o número de botões por inflorescência variou de 27 a 58 e média de 36 botões, enquanto o número de flores abertas por inflorescência foi de 5 a 45 flores abertas por dia.

Conforme Franco (1995), a disponibilidade de muitas flores abertas na inflorescência por dia é comum na família Fabaceae, sendo vantajosa para a espécie, pois aumenta a atratividade do polinizador, uma vez que há aumento na disponibilidade de néctar.

Com relação ao sistema de reprodução, Aken'ova \& Sumberg (1986) observaram que a autopolinização espontânea não ocorre nessa espécie, necessitando de agentes polinizadores para seu êxito reprodutivo. Considerando essa necessidade, objetivou-se, nesse trabalho, obter informações sobre a diversidade de abelhas visitantes na gliricídia, na região do Recôncavo Baiano, fornecendo informações sobre seus prováveis polinizadores.

O trabalho foi conduzido no campo experimental do Centro de Ciências Agrárias, Ambientais e Biológicas da Universidade Federal do Recôncavo da Bahia, localizada no município de Cruz das Almas, Bahia, situado no Recôncavo Baiano, a 12 40' 39" de latitude sul, 39 40' 23" de longitude oeste de Greenwich, altitude de $220 \mathrm{~m}$, temperatura média anual de $24,5^{\circ} \mathrm{C}$, umidade relativa de 80 $\%$ e precipitação pluvial média de $1224 \mathrm{~mm}$, segundo a classificação de Köppen, o clima é tropical quente úmido, AW a AM (ALMEIDA, 1999).

Os espécimes visitantes nas flores foram coletados durante o período de floração nos meses de setembro a novembro de 2005. Um total 15 inflorescências foram selecionadas, aleatoriamente, por intervalo de hora para a coleta efetiva das abelhas, utilizando um minuto por inflorescência, ao longo do intervalo de 07:00 às 18:00 horas.

Os insetos foram coletados com redes entomológicas, separados em morfoespécies e catalogados. A identificação dos espécimes foi baseada nos exemplares depositados no Museu Entomológico do Centro de Ciências Agrárias, Biológicas e Ambientais da UFRB.

Um total de 63 indivíduos foi coletado visitando as flores de gliricídia, distribuídos em três famílias, cinco tribos, oito gêneros e dez espécies (Tabela 1). A maior riqueza de espécies foi da família Apidae, destacando a subtribo Meliponina, com 50,79\% dos indivíduos. Dentre os gêneros, Xylocopa foi o que apresentou a maior riqueza de espécies.

Apis mellifera foi a espécie mais abundante, com freqüência relativa igual a 25,40\%, seguida de Trigona spinipes $(23,81 \%)$ e Nannotrigona testaceicornis $(14,28 \%)$.
Apis mellifera apresentou características favoráveis à dominância, como o hábito generalista de coleta, densidade populacional elevada das colônias perenes, sistemas sofisticados de comunicação, largo período diário de forrageamento, alta capacidade de enxamear, pouca exigência na escolha do local de nidificação, entre outras (ROUBIK, 1989).

A abundância de $T$. spinipes deve-se, entre outros aspectos, à versatilidade e a inacessibilidade dos locais para nidificação, à agressividade das operárias, ao número expressivo de espécies de flores onde as operárias coletam pólen e à falta de interesse do homem na exploração de seus produtos (ALMEIDA \& LOROCA, 1988).

A $N$. testaceicornis é uma espécie que está se destacandocom insetos visitantes na região do Recôncavo Baiano e que tem potencial como polinizador em determinadas culturas (MACHADO, 2006).

Levantamentos de abelhas visitantes de flores de fruteiras (CARVALHO et al., 1995) e leguminosas (CARVALHO \& MARQUES, 1995) na mesma região, também indicaram $A$. mellifera e $T$. spinipes como as mais freqüentes e abundantes.

Outros estudos realizados na região concluíram que $N$. testaceicornis é uma das espécies mais freqüentes em flores de Pimpinella anisum L. (BARROS et al., 2002) e de Helianthus annuus (MACHADO \& CARVALHO, 2006). Nesse último caso, A. mellifera e T. spinipes, também foram consideradas freqüentes. Em flores de Cajanus cajan Druce, Azevedo (2006) observou que T. spinipes foi a espécie mais abundante.

T. fuscipennis e X. grisescens apresentaram freqüência de $12,70 \%$, cada uma. T. fuscipennis também foi registrada visitando as flores de $C$. cajan (AZEVEDO, 2006) e de H. annuus (MACHADO \& CARVALHO, 2006).

Registros de espécies de Xylocopa como principais polinizadoras de G. sepium são citados por Aken’ova \& Sumberg (1986) na Nigéria, por Corbet \& Willmer (1980) nas Antilhas e por Janzen (1983) no México. No Brasil, Kill \& Drumond (2001) consideraram $X$. frontalis e $X$. grisescens como polinizadores efetivos de G. sepium.

As demais espécies de abelhas coletadas nas flores de G. sepium apresentaram freqüência abaixo de 3,5\% e são de ocorrência comum na região (BARROS et al., 2002; CARVALHO et al., 1995; CARVALHO \& MARQUES, 1995; MACHADO \& CARVALHO, 2006).

A distribuição dos indivíduos, por intervalo de hora, indica que o período da manhã foi o de maior atividade das abelhas, com pico de visitação no intervalo de 10:01 às 
Tabela 1 - Abelhas (Apoidea)* visitantes de gliricídia (Gliricidia sepium) no Recôncavo Baiano, no período de setembro a novembro de 2005.

\begin{tabular}{|c|c|c|}
\hline Família / Subfamília / Tribo / Subtribo / Espécie & $\begin{array}{c}\quad{ }^{\circ} \text { de } \\
\text { indivíduo }\end{array}$ & $\begin{array}{c}\text { Freqüência } \\
\text { Relativa (\%) }\end{array}$ \\
\hline \multicolumn{3}{|l|}{ Apidae } \\
\hline \multicolumn{3}{|l|}{ Apinae } \\
\hline \multicolumn{3}{|l|}{ Apini } \\
\hline \multicolumn{3}{|l|}{ Apina } \\
\hline Apis mellifera L. 1758 & 16 & 25,40 \\
\hline \multicolumn{3}{|l|}{ Meliponina } \\
\hline Nannotrigona testaceicornis (Lepeletier,1936) & 9 & 14,28 \\
\hline Trigona fuscipennis Friese, 1900 & 8 & 12,70 \\
\hline Trigona spinipes (Fabricius,1793) & 15 & 23,81 \\
\hline \multicolumn{3}{|l|}{ Centridini } \\
\hline Centris aenea Lepeletier, 1841 & 1 & 1,59 \\
\hline \multicolumn{3}{|l|}{ Xylocopinae } \\
\hline \multicolumn{3}{|l|}{ Xylocopini } \\
\hline Xylocopa frontalis (Olivier,1789) & 2 & 3,17 \\
\hline Xylocopa grisescens Lepeletier, 1841 & 8 & 12,70 \\
\hline Xylocopa suspecta Moure \& Camargo, 1988 & 2 & 3,17 \\
\hline \multicolumn{3}{|l|}{ Halictidae } \\
\hline \multicolumn{3}{|l|}{ Halictinae } \\
\hline \multicolumn{3}{|l|}{ Augochlorini } \\
\hline Augochlorella sp. & 1 & 1,59 \\
\hline \multicolumn{3}{|l|}{ Megachilidae } \\
\hline \multicolumn{3}{|l|}{ Megachilinae } \\
\hline \multicolumn{3}{|l|}{ Megachilini } \\
\hline Coelioxys sp. & 1 & 1,59 \\
\hline
\end{tabular}

"Classificação conforme Silveira et al. (2002).

11:00 horas (31,75\% dos indivíduos coletados) (Figura 1). Kill \& Drumond (2001), observaram que as abelhas visitaram mais intensamente as flores de G. sepium entre as 7:00 e 9:00 horas.

No período da tarde, ocorreu uma redução gradativa no número de indivíduos nas flores até as 18:00 horas. Segundo Kill \& Drumond (2001), a antese das flores de gliricídia é iniciada às 6:00 horas, permanecendo abertas até as 16:00 horas, quando é iniciado o processo de senescência floral, com o murchamento e a alteração da cor do estandarte.

A distribuição das espécies mais freqüentes nos intervalos de hora indica uma constância de ocorrência de
$88,82 \%$ para A. mellifera, $63,64 \%$ para $N$. testaceicornis e $45,45 \%$ para $T$. spinipes. Essa última espécie concentrou sua visita às flores no horário das 10:01 às 11:00 horas, com $67 \%$ dos indivíduos.

A preferência das abelhas por visitar as flores no horário da manhã, para a coleta de pólen e néctar na região, também foi observada por Barros et al. (2002), Carvalho et al. (2001) e Machado (2006).

Os resultados obtidos nas condições do presente estudo permitem concluir que $A$. mellifera, $T$. spinipes e $N$. testaceicornis são as espécies com potencial para a polinização das flores de gliricídias, na região do Recôncavo Baiano. 
世曹 Apis mellifera

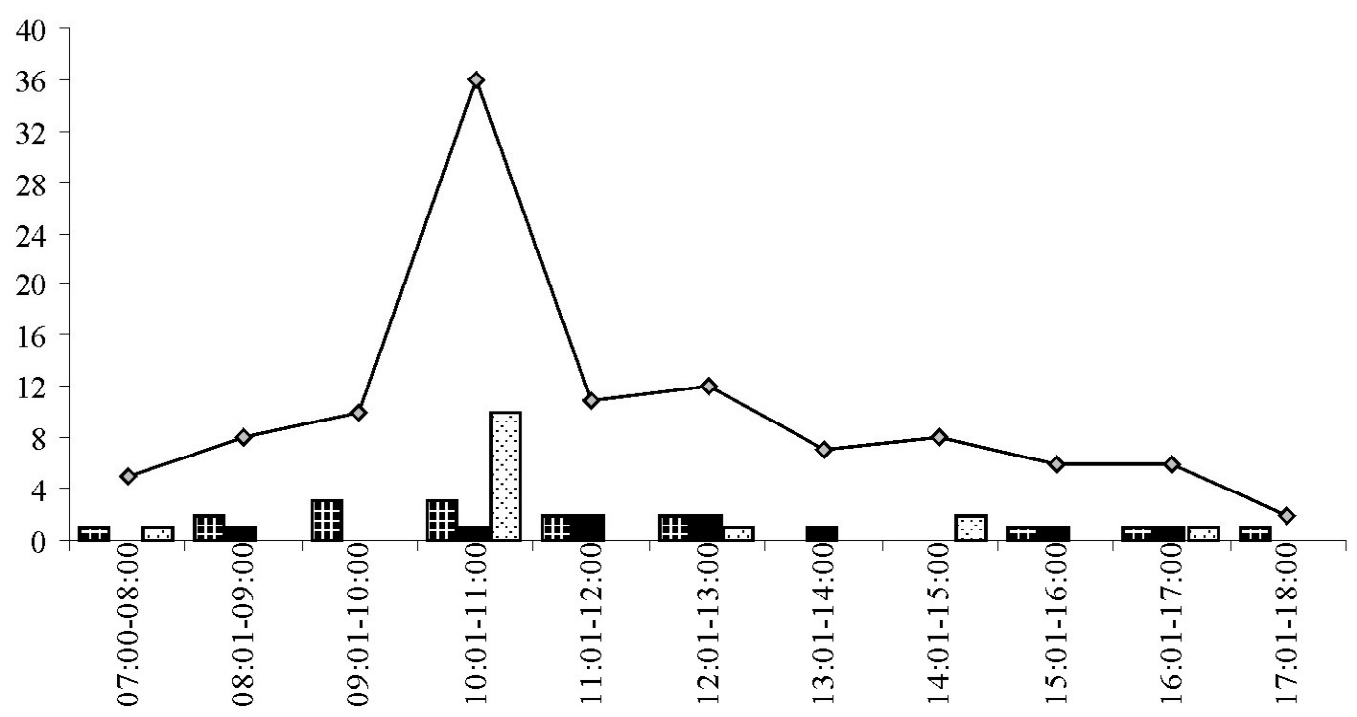

Figura 1 - Distribuição do número de indivíduos total e das três espécies de abelhas (Apoidea) mais freqüentes por intervalo de hora, em flores de gliricídia no Recôncavo Baiano.

\section{REFERÊNCIAS BIBLIOGRÁFICAS}

AKEN'OVA, M. E.; SUMBERG, J. E. Observations of the pollination systems of Gliricidia sepium. In: THAILAND INSTITUTE OF SCIENTIFIC AND TECHNOLOGICAL RESEARCH, 1986, Bangkok. Nitrogen fixing tree: research report. Bangkok: TISTR, 1986. v. 4, p. 29-30.

ALMEIDA, M. C. de; LAROCA, S. Trigona spinipes (Apidae, Meliponinae): taxonomia, bionomia e relação tróficas em áreas restritas. Acta Biológica Paranaense, Curitiba, v. 17, n. 1/4, p. 67-108, 1988.

ALMEIDA, O. A. Informações metereológicas do CNP: mandioca e fruticultura tropical. Cruz das Almas: Embrapa-CNPMF, 1999. 35 p. (Documentos, 34).

\section{AZEVEDO, R. L. Entomofauna associada ao feijão} guandu (Cajanus cajan (L.) Millspaugh) no Recôncavo Baiano. 2006. 45 f. Dissertação (Mestrado em Ciências Agrárias) - Universidade Federal da Bahia, Cruz das Almas, 2006.

BARROS, T. F. et al. Abelhas visitantes de flores de Pimpinella anisum L. Magistra, Cruz das Almas, v. 14, n. 1, p. 55-60, 2002.
CARVALHO, C. A. L. de; MARQUES, O. M. Abelhas (Hymenoptera, Apoidea ) em Cruz das Almas - BA: 2 espécies coletadas em leguminosas. Insecta, Cruz das Almas, v. 4, n. 2, p. 26-31, 1995.

CARVALHO, C. A. L. de; MARQUES, O. M.; SAMPAIO, H. S. V. Abelhas (Hymenoptera, Apoidea) em Cruz das Almas - BA: 1 espécies coletadas em fruteiras. Insecta, Cruz das Almas, v. 4, n. 1, p. 11-17, 1995.

CARVALHO, C. A. L. de; MARQUES, O. M.; VIDAL, C. A.; NEVES, A. M. S. Comportamento forrageiro de abelhas (Hymenoptera, Apoidea) em flores de Solanum palinacanthum Dunal (Solanaceae). Revista Brasileira de Zoociências, Juiz de Fora, v. 3, n. 1, p. 35-44, 2001.

COBERT, S. A.; WILLMER, P. G. Pollination of the yellow passion-fruit: nectar, pollen and carpenter bees. Journal of Agricultual Science, Cambridge, v. 95, n. 3, p. 655-666, 1980.

DRUMOND, M. A.; CARVALHO FILHO, O. M. de. Introdução e avaliação de Gliricidia sepium na região semi-árida do Nordeste Brasileiro. Disponível em: 4http ///www cpatsa.embrapa.br/servicos/catalogo/ ilivrorg/gliricidia.doc). Acesso em: 27 fev. 2006. 
FRANCO, A. L. M. Ecologia da polinização e biologia reprodutiva de sete espécies de Phaseoleae (Fabaceae). 1995. 123 f. Tese (Doutorado em Biologia Vegetal) Universidade Estadual de Campinas, Campinas, 1995.

JANZEN, D. H. Costa Rica natural history. Chicago: University of Chicago, 1983. 201 p.

KIILL, L. H. P.; DRUMOND, M. A. Biologia floral e sistema reprodutivo de Gliricidia sepium (Jacq.) Steud. (Fabaceae-Papilionoidae) na Região de Petrolina, Pernambuco. Ciência Rural, Londrina, v. 31, n. 4, p. 597601, 2001.

MACHADO, C. S. Aspectos de interesse da polinização entomófila de Helianthus annuus L. no Recôncavo Baiano. 2006. 44 f. Dissertação (Mestrado em Ciências Agrárias) - Universidade Federal da Bahia, Cruz das Almas, 2006.
MACHADO, C. S.; CARVALHO, C. A. L. de. Abelhas (Hymenoptera: Apoidea) visitantes dos capítulos de girassol no Recôncavo Baiano. Ciência Rural, Londrina, v. 36, n. 5, 2006.

NEVES, M. C. P. Gliricídia. Disponível em: <http:// www.cnpab.embrapa.br/index.htmis. Acesso em: $27 \mathrm{fev}$. 2006.

ROUBIK, D. W. Ecology and natural history of tropical bees. Cambridge: Cambridge University, 1989. 514 p.

SILVEIRA, F. A.; MELO, G. A. R.; ALMEIDA, E. A. B. Abelhas brasileiras: sistemática e identificação. Belo Horizonte: Composição e Arte, 2002. 253 p.

SUMBERG, J. E. Note on flowering and seed production in a yong Gliricidia sepium seed orchard. Tropical Agriculture, Trinidad, v. 62, n. 1, p. 17-24, 1985. 